\title{
The European Union standards for tuberculosis care: do they need an update?
}

\author{
Marieke J. van der Werf ${ }^{1}$, Andreas Sandgren ${ }^{1}$, Lia D’Ambrosioํ․ Francesco Blasi ${ }^{3}$ \\ and Giovanni Battista Migliori²
}

Affiliations: 'European Centre for Disease Prevention and Control (ECDC), Stockholm, Sweden. ${ }^{2}$ WHO Collaborating Centre for TB and Lung Diseases, Fondazione S. Maugeri, Care and Research Institute, Tradate, and ${ }^{3}$ Dipartimento Fisiopatologia Medico-Chirurgica e dei Trapianti, University of Milan, IRCCS Fondazione Cà Granda, Milan, Italy.

Correspondence: G.B. Migliori, WHO Collaborating Centre for TB and Lung Diseases, Fondazione Salvatore Maugeri, Care and Research Institute, via Roncaccio 16, 21049, Tradate, Italy. E-mail: giovannibattista.migliori afsm.it

-

@ERSpublications

Differences between ESTC and new ISTC discussed to inform clinicians and public health officers on best TB management http://ow.ly/tgbzt

The International Standards for Tuberculosis Care (ISTC), published for the first time in 2006 [1], introduced a new way of looking at clinical and public health guidelines for tuberculosis (TB). The European Respiratory Journal published an editorial explaining the ISTC, promoting the rapid uptake of the standards [2].

While guidelines represent a long, comprehensive document containing all the details the physician might need for managing $\mathrm{TB}$, the standards are a simple set of 21 principles that guide day-to-day clinical decisions $[3,4]$. In other words, guidelines are usually available in a physician's library while the standards are kept on their desk in the office.

The ISTC prescribe a widely accepted level of TB care which guides all healthcare providers and clinicians, both public and private, in achieving optimal standards in managing patients who have, or are suspected of having, active TB [1-4].

The European Centre for Disease Prevention and Control (ECDC) and the European Respiratory Society (ERS) jointly developed a European Union (EU) adaptation of the ISTC document, known as the European Union Standards for Tuberculosis Care (ESTC) [4]. These standards are tailored to the specific context of the EU/European Economic Area (EEA) as follows [4, 5].

1) Although the majority of EU/EEA member states have a low incidence of TB, it is a heterogeneous setting with some countries having a high or intermediate level of $\mathrm{TB}$, with varying levels of multidrug-resistant (MDR)-TB and TB-HIV co-infection, and some countries bordering non-EU countries with a higher TB and MDR-TB burden.

2) TB services are fully integrated and merged within the health system in the majority of EU/EEA member states. This presents peculiarities in allocating responsibilities for the delivery of TB care.

3) The EU/EEA member states have a long established tradition of TB control that has evolved over the past decades. Implementation of new tools and high standards of diagnosis and care is aimed for in the EU/EEA member states.

Received: Dec 122013 | Accepted after revision: Jan 092014

Conflict of interest: Disclosures can be found alongside the online version of this article at www.erj.ersjournals.com 
4) Several EU/EEA member states are pursuing TB elimination, sharing a common platform (based on the Wolfheze documents [6] and the Framework Action Plan to Fight TB in the EU [7]) with the ECDC and World Health Organization (WHO)-coordinated TB surveillance network and system [6-9].

The standards are designed as a living document that will be revised as technology, resources and circumstances change. Also, they complement existing national or international guidelines, being consistent with the WHO definitions and recommendations [5]. The ISTC were revised in 2009, while a new third edition has recently been presented to commemorate the 2014 World TB Day [3]. This prompted the ECDC and the ERS to compare the third edition of the ISTC and the ESTC to see what the differences are and to evaluate if a revision of the ESTC is necessary.

The core ECDC and ERS experts who coordinated the effort of the expert group developing the ESTC made a careful comparison of the new ISTC and ESTC standards (table 1), underlining the content that was not shared by both documents and summarising the differences. This will allow clinicians and public health officers to rapidly capture the innovative elements included in the third edition of the ISTC and understand to what extent they can be applied in the EU/EEA setting.

In terms of structure, the ESTC (consistent with the second edition of the ISTC [16]) contained 21 standards organised into four sections: 1) standards for diagnosis; 2) standards for treatment; 3) standards for addressing HIV infection and other comorbid conditions; and 4) standards for public health.

A new standard has been included in the third edition of the ISTC [3], to make clinicians aware that specific risk groups exist and that TB has a higher prevalence in these risk groups than in the general population. This issue is tackled by the ESTC in standards 1 and 4 (table 1).

No relevant differences were found between the ESTC and ISTC in standards 9, 13, 14, 17, 19, 20 and 21.

In standard 1, the ESTC take into account the variability of signs and symptoms TB presents with, while the ISTC make reference to chronic cough as the global "pivotal" symptom traditionally allowing investigation of a patient for TB. It seems adequate to describe the reality of the EU setting in the ESTC where chronic cough is more related to smoking and other lung diseases.

In standard 2, while the ISTC recommend Xpert MTB/RIF as the first choice for diagnosis (consistent with the present WHO policy to promote the wide peripheral use of the test [10-12]), the ESTC mention rapid testing for the identification of rifampicin and isoniazid resistance using validated tools. This makes sense in the EU, given the focus is on quality-assured culture-based methods and easy access to drug susceptibility testing (DST). The ESTC does not yet include a negative recommendation on blood-based serological tests since this WHO recommendation was published after the launch of the ESTC [13]. The WHO recommendation is for the use of interferon- $\gamma$ release assays (IGRAs) in low- and middle-income countries [14]. Specific guidance on the use of IGRAs in the EU is provided by the ECDC [17-19]. In the perspective of TB elimination, IGRAs might gain additional interest in the diagnosis of TB infection.

Similarly, in standard 3 the ESTC advocate the use of culture and DST in extrapulmonary TB, while the ISTC consistently recommend the use of Xpert MTB/RIF.

In standard 5, the ESTC provide guidance on how to diagnose culture-negative TB, while the ISTC focus on smear-negative TB. For diagnosing culture-negative TB, a trial of broad-spectrum antimicrobials may be used according to the ESTC standard, such trial regimens should not include fluoroquinolones. The ESTC specifically recommend expedited investigation of immunocompromised patients, who represent a priority in the EU setting.

Standard 6 of the ISTC indicates that along with sputum smear microscopy and culture, the Xpert MTB/RIF test can also be used for bacterial confirmation of TB in children. The corresponding ESTC standard provides more details on how to diagnose TB in children.

In standard 7, the ESTC underline the need for the treating physician to collaborate with the local public health and/or community health services to perform a contact investigation [20].

Standard 8, apart from the different wording (internationally recommended treatment regimens in ESTC versus WHO recommended regimens in ISTC), is consistent in the two documents. The ESTC recommends the use of fixed-dose combinations.

In standard 10, the ISTC is more prescriptive on what test to use for DST when the sputum is still positive at 3 months, i.e. Xpert MTB/RIF should be used.

In standard 11, the ISTC is more restrictive in recommending DST. Instead of recommending it for all TB patients, as in the ESTC, the ISTC recommend DST for previously treated patients, patients who remain 


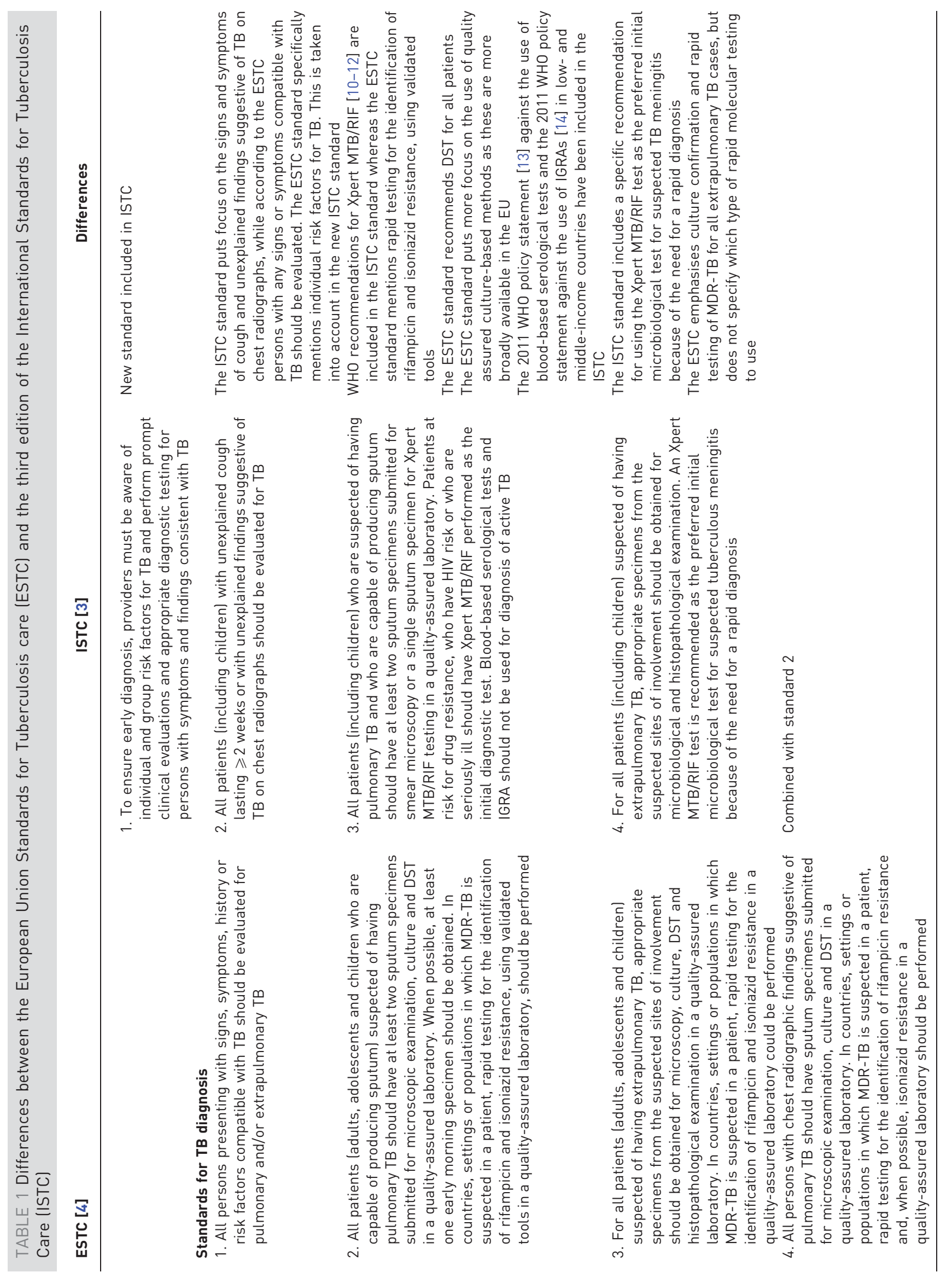




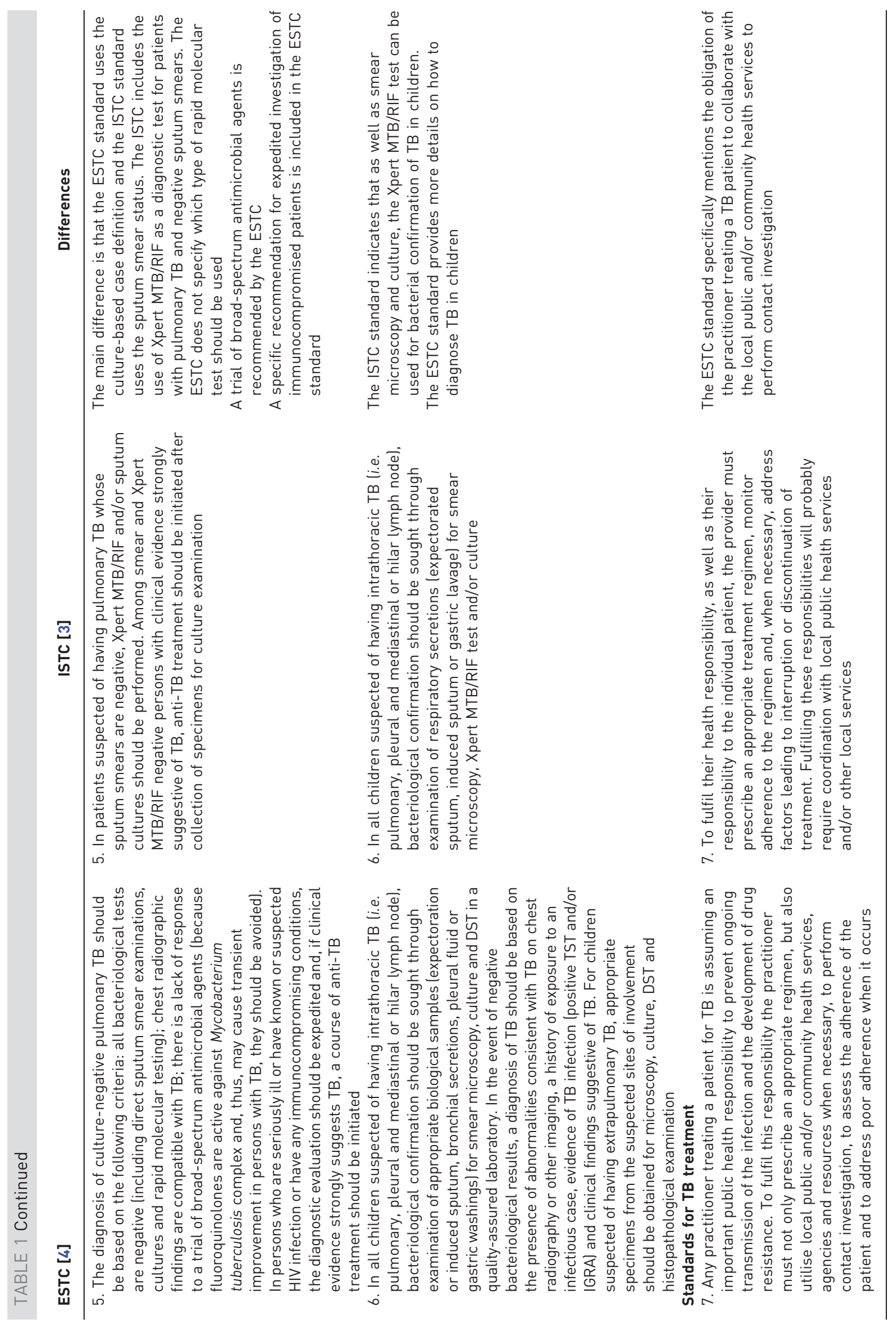




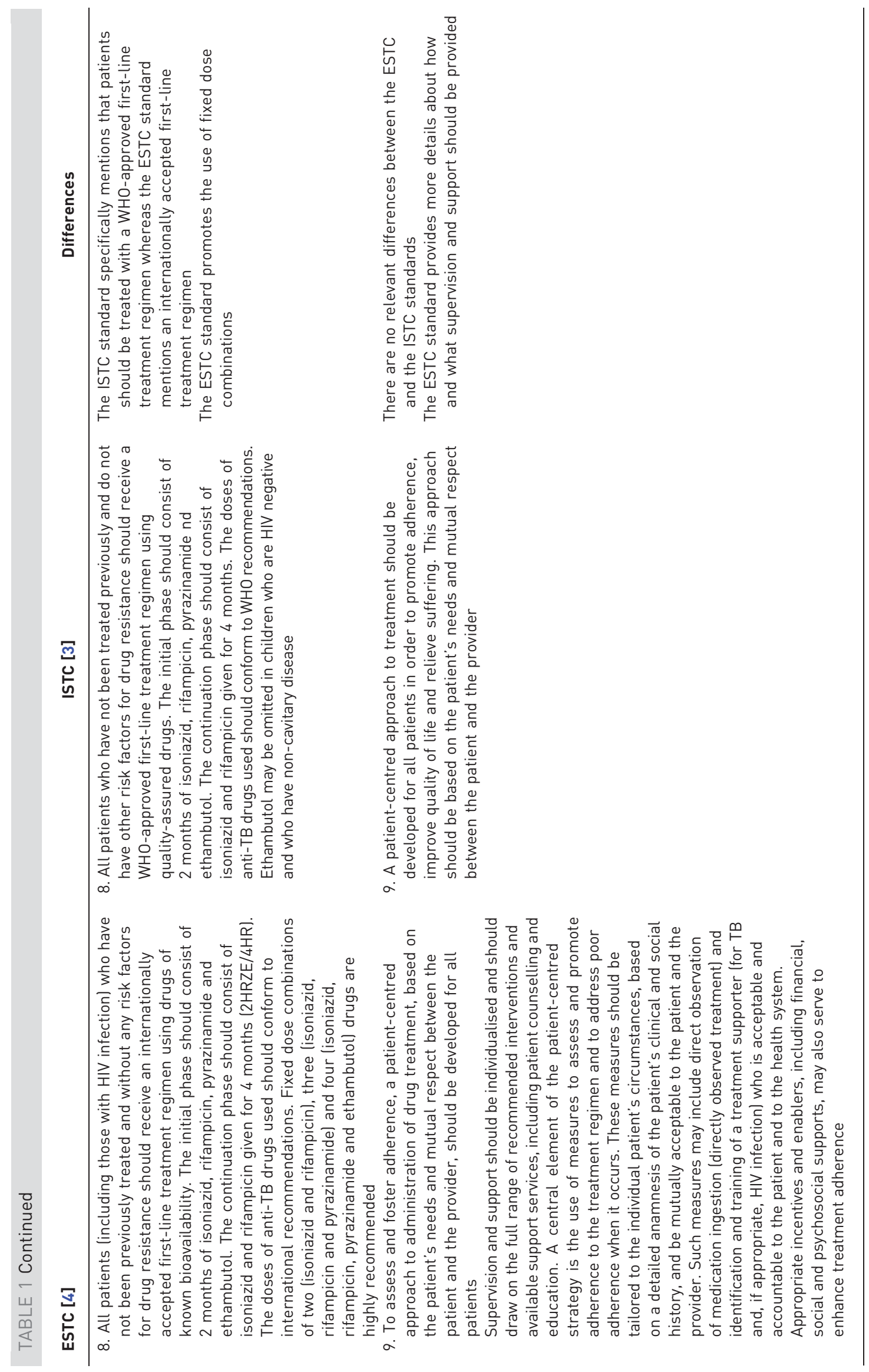




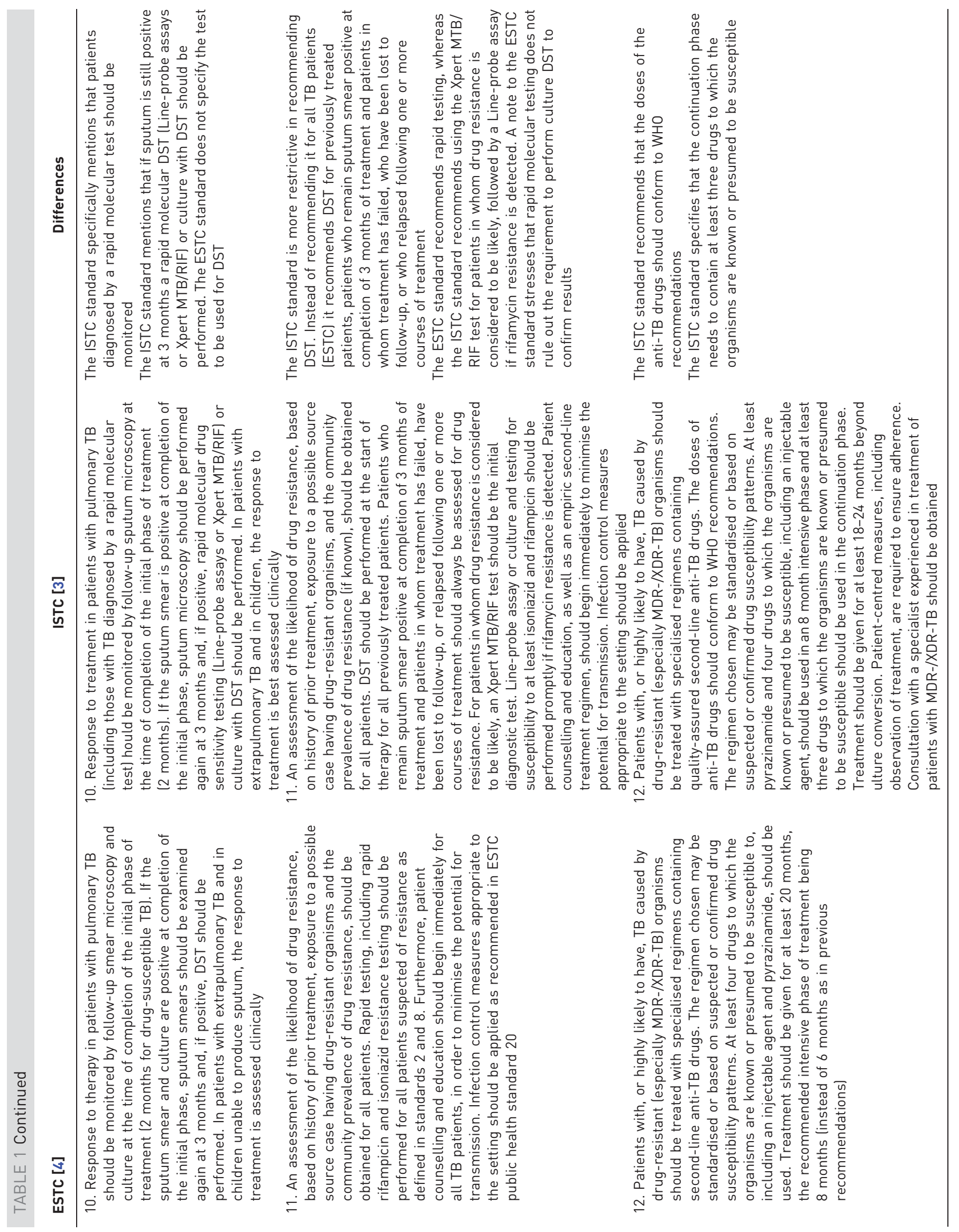




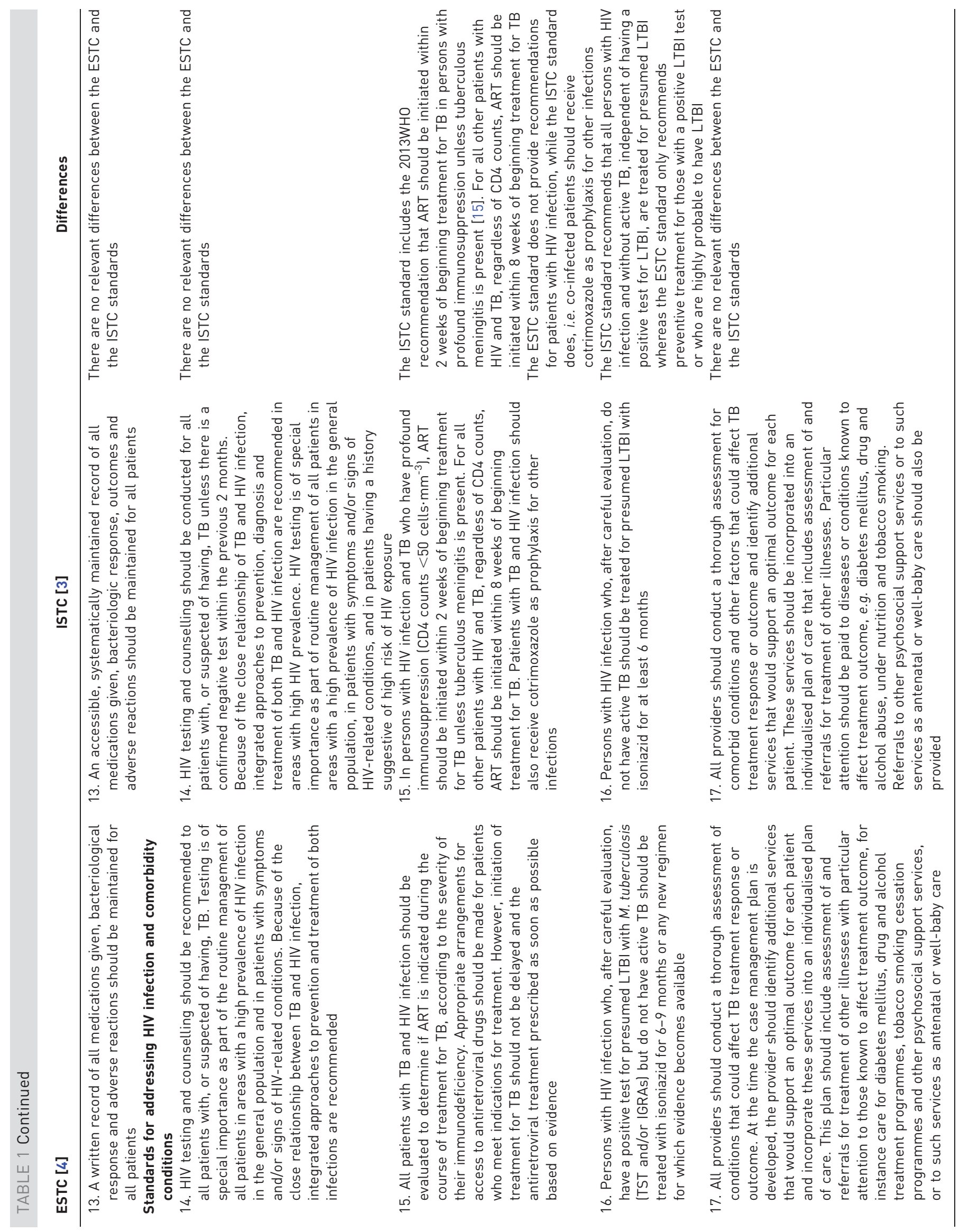



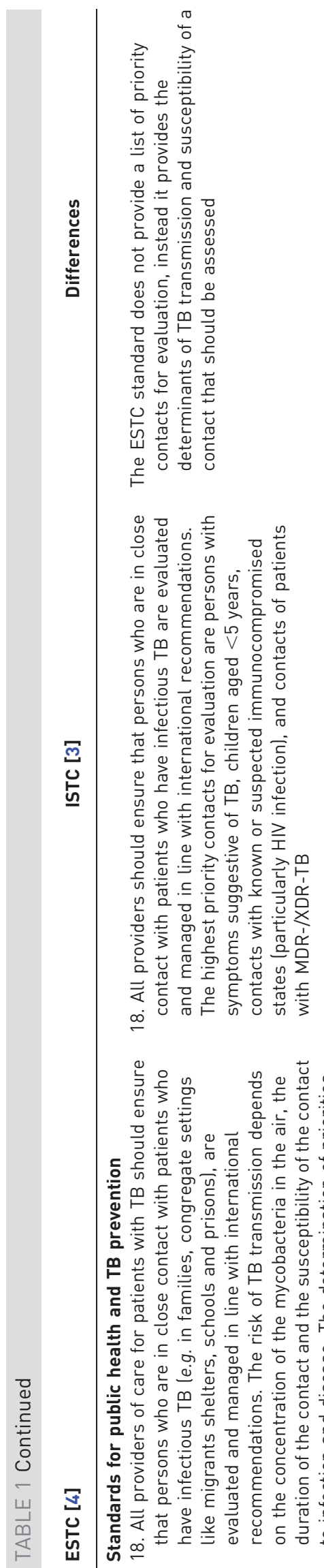

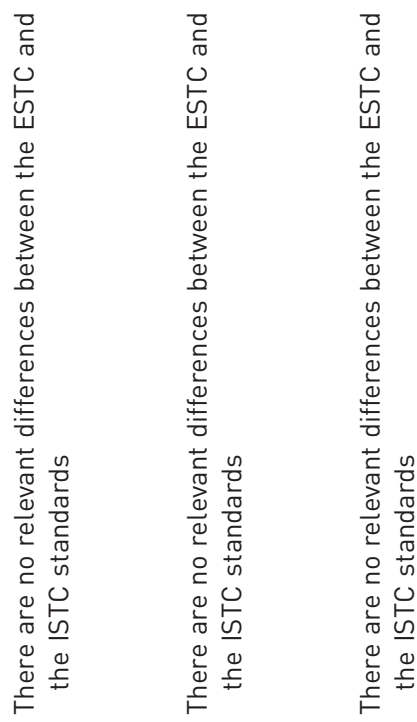

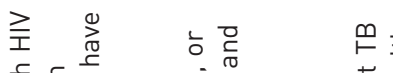

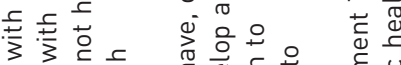

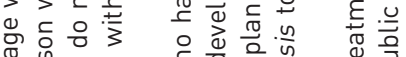

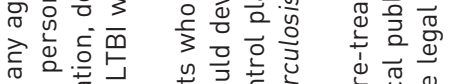

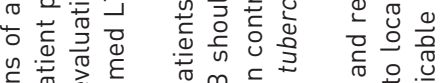

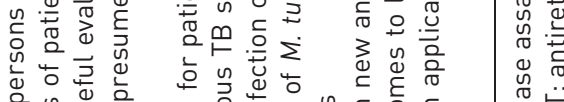

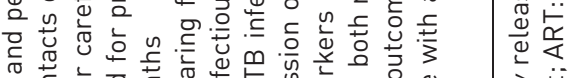

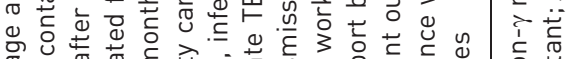

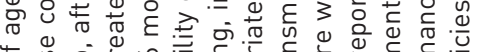

to

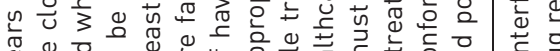

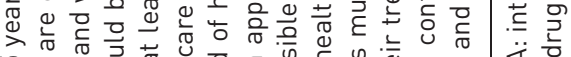

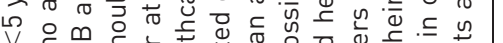

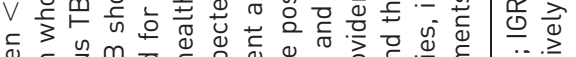

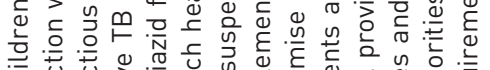

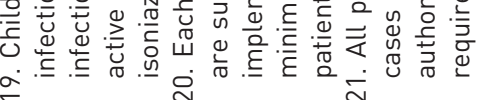

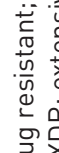

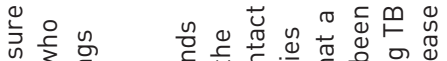

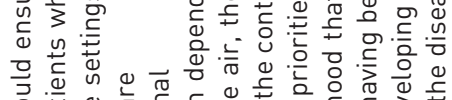

ธ ธ

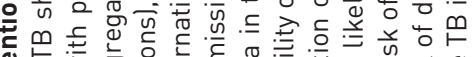

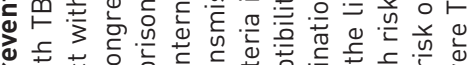

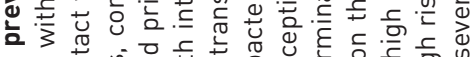

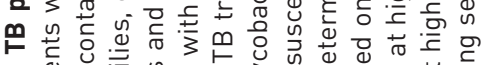

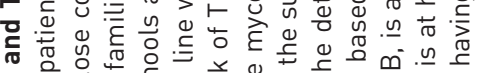

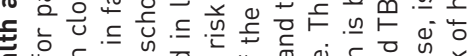

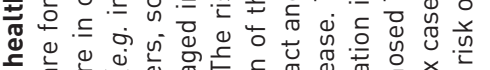

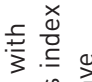

亡。

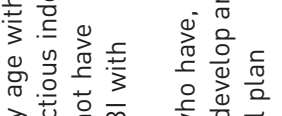

$\stackrel{\oplus}{\bullet}$

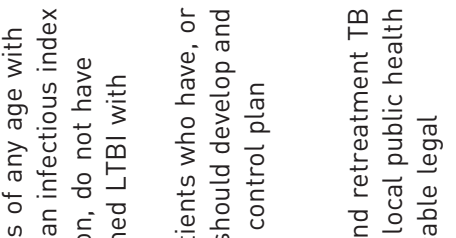

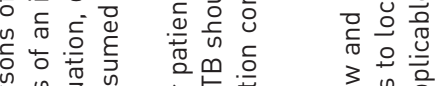

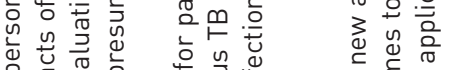

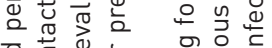

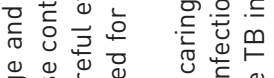

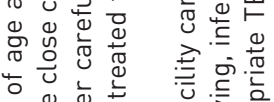

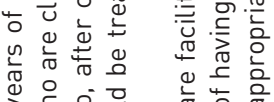

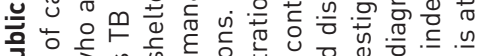

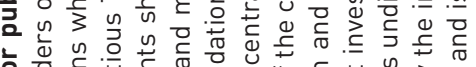

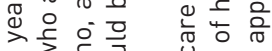

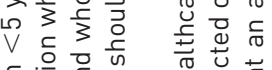

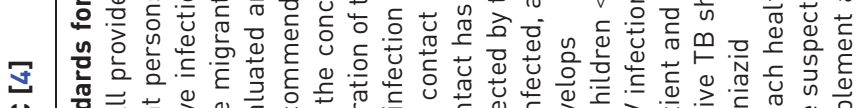

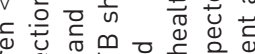

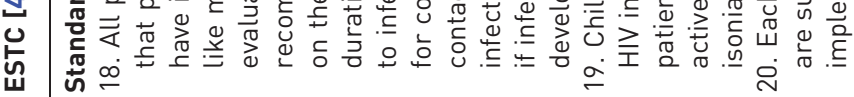

告

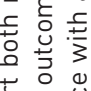

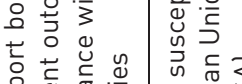

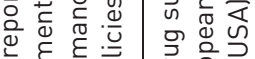

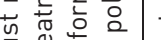

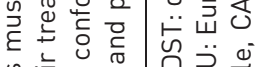

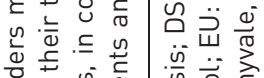

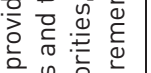

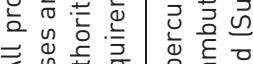

¿

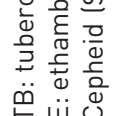


sputum smear positive at completion of 3 months of treatment, and patients in whom treatment has failed, patients lost to follow-up, or patients who relapsed following one or more treatment courses.

In standard 12 the recommendations are quite similar. The ESTC contain an EU-adapted supplement focusing on the need to treat MDR-TB cases in specialised settings with individualised and DST-based regimens, and under the guidance of a panel of experts [21-24].

The ISTC standard 15 includes the recent WHO recommendations to initiate antiretroviral treatment within 2 weeks of beginning TB treatment in HIV-infected individuals with profound immunosuppression or within 8 weeks for all others [15]. The ESTC specifies that patients with TB and HIV should be evaluated to determine if antiretroviral therapy is indicated.

The main difference between the ISTC and the ESTC in standard 16 is that the ISTC recommend treatment of latent TB infection for all HIV-infected persons who do not have active TB whereas the ESTC only recommend it for those either infected or likely to be infected by Mycobacterium tuberculosis.

In standard 18 the ISTC provides a specific list of priority contacts for contact evaluation, whereas the ESTC states which determinants of TB transmission and susceptibility of a contact should be evaluated.

The analysis of the differences between the two documents (table 1) shows the consistency of their recommendations given the different setting (EU versus global) in which they are applied.

As new evidence is rapidly growing, a revision of the ESTC will be appropriate in about 2 years. The critical elements that will require modification will be represented, in our opinion, by the following factors: 1) the WHO post-2015 strategy, which will emphasise elimination and will pose new targets and milestones; 2) the progressive implementation of the ECDC elimination framework vis-à-vis the evidence on how the interventions necessary to reach elimination are applied in the $\mathrm{EU}[6,25,26]$; and 3) the introduction of new drugs and new regimens (potentially involving delamanid, bedaquiline and PA-824) to treat MDR-TB, but also potentially drug-susceptible TB and latent TB infection [27-30].

We hope that the comparative analysis of the two documents will further contribute to help clinicians in making the correct diagnosis and in the undertaking the correct treatment and public health actions, to ensure the best management of TB and MDR-TB cases.

\section{Acknowledgements}

We wish to thank R. Centis (WHO Collaborating Centre for TB and Lung Diseases, Fondazione S. Maugeri, Care and Research Institute, Tradate, Italy) for her suggestions and technical support, and P. Hopewell and F. Du Melle (American Thoracic Society, New York, NY, USA) for ensuring consistency of this document with the final version of the International Standards for Tuberculosis Care.

\section{References}

1 Tuberculosis Coalition for Technical Assistance. International Standards for Tuberculosis Care (ISTC). Tuberculosis Coalition for Technical Assistance, The Hague, 2006.

2 Migliori GB, Hopewell PC, Blasi F, et al. Improving the TB case management: the International Standards for Tuberculosis Care. Eur Respir J 2006; 28: 687-690.

3 Tuberculosis Coalition for Technical Assistance. International Standards for Tuberculosis Care (ISTC). 3rd Edn. Tuberculosis Coalition for Technical Assistance, The Hague, (in press).

4 Migliori GB, Zellweger JP, Abubakar I, et al. European Union Standards for Tuberculosis Care. Eur Respir J 2012; 39: $807-819$

5 Migliori GB, Sotgiu G, Blasi F, et al. Towards the development of EU/EEA Standards for Tuberculosis Care (ESTC). Eur Respir J 2011; 38: 493-495.

6 Veen J, Migliori GB, Raviglione M, et al. Harmonisation of TB control in the WHO European region: the history of the Wolfheze Workshops. Eur Respir J 2011; 37: 950-959.

7 European Centre for Disease Prevention and Control. Framework action plan to fight tuberculosis in the European Union. ECDC, Stockholm, 2008.

8 Hollo V, Zucs P, Kodmon C, et al. Marking 15 years of efforts towards a comprehensive European TB surveillance system: the epidemiological situation of TB in the EU/EEA in 2009. Euro Surveill 2011; 16: 19822.

9 D’Ambrosio L, Dara M, Tadolini M, et al. Tuberculosis elimination: theory and practice in Europe. Eur Respir J 2013 [in press DOI: 10.1183/09031936.00198813].

10 World Health Organization. Automated Real Time Nucleic Acid Amplification Technology for Rapid and Simultaneous Detection of Tuberculosis and Rifampicin Resistance: Xpert MTB/RIF System. Policy statement. WHO, Geneva, 2011.

11 World Health Organization. Tuberculosis Diagnostics Xpert MTB/RIF Test. www.who.int/tb/publications/ Xpert_factsheet.pdf Date last accessed: February 6, 2014. Date last updated: October 2013.

12 Weyer K, Mirzayev F, Migliori GB, et al. Rapid molecular TB diagnosis: evidence, policy making and global implementation of Xpert MTB/RIF. Eur Respir J 2013; 42: 252-271.

13 World Health Organization. Commercial Serodiagnostic Tests for Diagnosis of Tuberculosis. Policy statement. WHO, Geneva, 2011.

14 World Health Organization. Use of Tuberculosis Interferon Gamma Release Assays (IGRAs) in Low and Middle Income Countries. Policy statement. WHO, Geneva, 2011. 
15 World Health Organization. WHO policy on collaborative TB/HIV activities. Guidelines for national programmes and other stakeholders. WHO, Geneva, 2012.

16 Tuberculosis Coalition for Technical Assistance. International Standards for Tuberculosis Care (ISTC). 2nd Edn. Tuberculosis Coalition for Technical Assistance, The Hague, 2009.

17 European Centre for Disease Prevention and Control. Use of interferon-gamma release assays in support of TB diagnosis. ECDC, Stockholm, 2011.

18 Sester M, Sotgiu G, Lange C, et al. Interferon- $\gamma$ release assays for the diagnosis of active tuberculosis: a systematic review and meta-analysis. Eur Respir J 2011; 37: 100-111.

19 Diel R, Goletti D, Ferrara G, et al. Interferon- $\gamma$ release assays for the diagnosis of latent Mycobacterium tuberculosis infection: a systematic review and meta-analysis. Eur Respir J 2011; 37: 88-99.

20 Erkens CGM, Kamphorst M, Abubakar I, et al. Tuberculosis contact investigation in low prevalence countries: a European consensus. Eur Respir J 2010; 36: 925-949.

21 Blasi F, Barnes PJ, Gaga M, et al. Future directions for the ERS: Presidential plans. Eur Respir J 2013; 42: 875-880.

22 Blasi F, Dara M, van der Werf MJ, et al. Supporting TB clinicians managing difficult cases: the ERS/WHO Consilium. Eur Respir J 2013; 41: 491-494.

23 Blasi F, Reichman LB, Migliori GB. Presenting the European Forum for TB Innovation: innovative thinking in progressing towards TB elimination in Europe. Eur Respir J 2012; 40: 806-808.

24 D'Ambrosio L, Tadolini M, Dupasquier S, et al. ERS/WHO Tuberculosis Consilium: reporting on initial 10 cases. Eur Respir J 2014; 43: 286-289.

25 European Centre for Disease Prevention and Control. Progressing towards TB elimination. A follow-up to the Framework Action Plan to Fight Tuberculosis in the European Union. ECDC, Stockholm, 2010.

26 Diel R, Loddenkemper R, Zellweger JP, et al. Old ideas to innovate TB control: preventive treatment to achieve elimination. Eur Respir J 2013; 42: 785-801.

27 Diacon AH, Dawson R, von Groote-Bidlingmaier F, et al. The 14-day bactericidal activity of combinations of PA824, bedaquiline, pyrazinamide and moxifloxacin: the path to novel antituberculosis treatment regimens. Lancet 2012; 380: 986-993.

28 Migliori GB, Sotgiu G. Treatment of tuberculosis: have we turned the corner? Lancet 2012; 380: 955-957.

29 Skripconoka V, Danilovits M, Pehme L, et al. Delamanid improves outcomes and reduces mortality in multidrugresistant tuberculosis. Eur Respir J 2013; 41: 1393-1400.

30 Tiberi S, De Lorenzo S, Centis R, et al. Bedaquiline in MDR-/XDR-TB cases: first experience on compassionate use. Eur Respir J 2014; 43: 289-292. 